\title{
Effect of extrinsic noise on vibrotactile information processing channels
}

\author{
RONALD T. VERRILLO and ANTHONY J. CAPRARO \\ Institute for Sensory Research, Syracuse University, Merrill Lane, Syracuse, New York 13210
}

\begin{abstract}
Cutaneous receptors sensitive to high-frequency vibration produce spatial summation at threshold levels of stimulation, whereas the response of the receptor population sensitive to low frequencies appears to be independent of contactor size at threshold. Reports have been made of a phenomenon in which the presumably nonsummating population of mechanoreceptors produce spatial summation at suprathreshold levels. A possible explanation concerns the relative signal-to-noise ratios of cutaneous mechanoreceptor systems. By selectively masking the systems with narrow-band and wide-band noise, it can be shown that any combination of signal frequency, masking noise, and contactor size that predominantly activates either system results in a linear effect of the masker on the threshold of the signal. Combinations of the experimental variables that activate both receptor systems simultaneously reduce the effect of the masking by one-half. This is interpreted as evidence of an interactive process between receptor systems within the central nervous system. The effects of signal-to-noise ratio and intrasensory interactions are discussed.
\end{abstract}

The vibrotactile threshold of detectability decreases at an approximate rate of $3.0 \mathrm{~dB}$ per doubling of the contactor area at frequencies above $40 \mathrm{~Hz}$, indicating a near perfect summation of energy over space, whereas thresholds for low frequencies (below $40 \mathrm{~Hz}$ ) appear to be independent of contactor size (Verrillo, 1963, 1966a). The dependence of spatial summation on stimulus frequency has been interpreted as an indication that more than one type of cutaneous mechanoreceptive 'system is involved in vibratory sensation; a low-frequency system not capable of producing spatial summation and a high-frequency system which summates energy over space. Rather strong evidence suggests that Pacinian corpuscles make up the receptor population capable of spatial summation (Verrillo, 1966b, c, 1968). This evidence was later corroborated by direct neurophysiological experiments (Talbot, Darian-Smith, Kornhuber, \& Mountcastle, 1968). Subsequent psychophysical and neurophysiological experimentation has left little doubt that vibrotaction is subserved by more than one mechanoreceptive end organ and that spatial summation is a property of the Pacinian corpuscle population (Harrington \& Merzenich, 1970; Johnson, 1974; Merzenich \& Harrington, 1969).

An apparent paradox has been reported in a recent publication (Verrillo, 1974) showing that, while

This research was supported by Grant NS-09940 from the National Institutes of Health, United States Department of Health, Education and Welfare. The authors are indebted to Dr. Jozef Zwislocki for the hypothesis that led to the design of these experiments and are grateful for his continued interest as the experiments progressed. The authors also express their appreciation to Dr. Earl Kletsky for his patient help with all aspects of calibrating the equipment for determining noise levels. spatial summation does not occur at threshold levels for low-frequency stimuli, it does occur at suprathreshold levels. Subjective-magnitude curves were determined by the method of numerical magnitude balance at $25 \mathrm{~Hz}$ for a large and a small contactor. Although the subjective-intensity functions originated from a common point in the vicinity of threshold, the curves for the two contactor sizes diverged at suprathreshold levels. This indicates that the summation of energy over space began to occur at intensities very near to the threshold of detectability. The separation of the curves increased with intensity, and in the vicinity of $15 \mathrm{~dB}$ SL it reached a value of approximately 7 to $9 \mathrm{~dB}$. Green and Craig (1974) have since reported spatial summation for low frequencies $(25$ and $40 \mathrm{~Hz}$ ) at suprathreshold levels. They attribute the summation simply to mechanical coupling factors between the contactor and skin, which affect the intensity range at which the Pacinian corpuscles become entrained. Figure 1 compares the data of Green and Craig with those of Verrillo (1974), showing the difference in amplitude level between two contactor sizes as a function of sensation level. Amplitude level is defined as the ratio of a vibration amplitude, expressed in decibels, to a reference amplitude of 1.0 micron of peak displacement. A high degree of similarity in the two sets of data is apparent, although Verrillo used direct scaling and Green and Craig used a matching procedure. The figure shows low-frequency spatial summation increasing with amplitude at suprathreshold levels. At high frequencies, the amount of summation remains constant at both threshold and suprathreshold levels. Near threshold, the summation is consistent with results previously reported (Verrillo, 1963). Green 


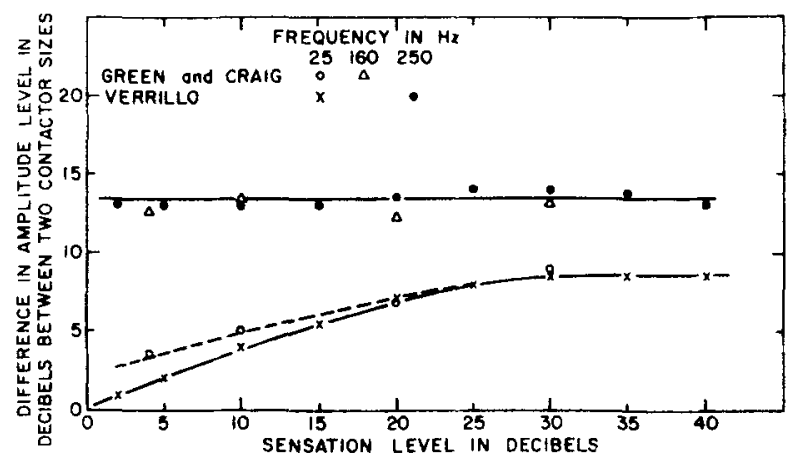

Figure 1. Comparison of data obtained by Green and Craig (1974) with that of Verrillo (1974) showing the difference in amplitude level between two contactor sizes as a function of sensation level. The amount of spatial summation is shown in the difference in amplitude level between the two contactor sizes. At high frequencies (Green \& Craig, $160 \mathrm{~Hz}, \triangle$; Verrillo, $250 \mathrm{~Hz}$, •), the spatial summation is constant at threshold and suprathreshold levels. At $25 \mathrm{~Hz}$ (Green \& Craig, $O$; Verrillo, $x$ ), summation is absent at threshold and increases with amplitude above threshold.

and Craig obtained a slightly greater summation for $25 \mathrm{~Hz}$ at low sensation levels. Our result, obtained by the method of numerical magnitude balance, showing no difference in amplitude level between the two contactor sizes at threshold, is consistent with previous results (Verrillo, 1963, 1968).

The aim of the current experiments was to investigate further the phenomenon of suprathreshold spatial summation in a population of mechanoreceptors presumed to be nonsummating. A series of masking experiments was performed in which physical parameters were selected that stimulate the two neural populations selectively and nonselectively. The effect was investigated by measuring vibrotactile thresholds in the presence of a variety of masking noises.

\section{APPARATUS AND PROCEDURES}

The apparatus used in the experiments has been described in detail in a previous publication (Verrillo, 1963). It consisted of a Goodmans $390 \mathrm{~A}$ vibrator mounted on the platen of a drill press assembly that provided fine adjustments in the position of the vibrator. The subject sat next to the assembly with his forearm and hand resting comfortably on a rigid surface located above the vibrator. The contactor protruded into a hole in the rigid surface to make contact with the surface of the subject's thenar eminence. The gap between the contactor and the rigid surround was $1.0 \mathrm{~mm}$, and the vibrator height was adjusted so that the contactor pressed $0.5 \mathrm{~mm}$ into the thenar eminence of the right hand. Subjects wore earphones which delivered narrow-band noise to mask airborne sounds emitted by the vibrator. A carefully calibrated Endevco accelerometer mounted on the moving element of the vibrator was used to monitor the amplitude of vibration. The entire assembly was located inside a booth that excluded unwanted sounds and vibrations.

Sinusoidal signals were produced by a Hewlett-Packard $200 \mathrm{CDR}$ sine-wave generator. The random-noise maskers were provided by a General Radio 1381 random-noise generator. Different bandwidths of noise were produced by passing the output of the noise generator through an Allison $2 \mathrm{ABR}$ variable filter. The following bandwidths of noise were used: wide band, 2.0 to $4,800 \mathrm{~Hz}$; narrow band/low, 15 to $45 \mathrm{~Hz}$; and narrow band/high, 240 to $270 \mathrm{~Hz}$. Experimental results (see Figure 3) showed that the wide-band noise was effective in producing a response in both high- and low-frequency populations. Signals from the sine wave and noise channels were mixed with a Kepco BOP $36-5(\mathrm{M})$, used as a power amplifier, before delivery to the vibrator.

Thresholds were obtained in the presence of maskers from three highly trained subjects at two frequencies $(25$ and $250 \mathrm{~Hz})$ and two contactor sizes $\left(0.28\right.$ and $\left.2.9 \mathrm{~cm}^{2}\right)$ using a Békésy recording attenuator. The time sequence of the sinusoids was $600 \mathrm{msec}$ ON and $1,400 \mathrm{msec}$ OFF with a $50-\mathrm{msec}$ rise/fall time. The maskers were $O N$ continuously during each threshold measu rement. Signals and maskers were delivered simultaneously through the same contactor. Each threshold measurement was based on a 2.0 -min final testing period containing an average of 12 ascending and 12 descending excursions. Examination of the response consistency within the 2.0 -min periods indicate that adaptation to the noise maskers was not a factor in the results.

In order to establish the spatial effects of the maskers alone, thresholds were determined for bursts of noise at each of the band wid ths used in the masking experiment with contactor areas of $0.02,0.34,0.72$, and $2.9 \mathrm{~cm}^{2}$. For these measurements, the time sequence was the same as that used for the sinusoids in the masking experiments.

Threshold values for sinusoidal signals are expressed in decibels referred to 1.0 micron of peak displacement. The intensities of the masking noises are expressed in sensation level (SL) or decibels referred to 1.0 micron RMS displacement.

\section{RATIONALE}

Several hypotheses have been proposed to explain the production of spatial summation at suprathreshold levels in a presumably nonsummating population of mechanoreceptors. Green and Craig (1974) have suggested that spatial summation at low frequencies is produced by the activation of Pacinian corpuscles by high-intensity stimuli. In Figure 2, both the psychophysical and physiological frequency response curves of the Pacinian system are shown. The Pacinian system is responsible for the U-shaped portion of the psychophysical curve (solid circles) which was obtained using a $2.9-\mathrm{cm}^{2}$ contactor (Verrillo, Fraioli, \& Smith, 1969). At low frequencies, the flat response is determined by the non-Pacinian receptors. The low-frequency portion of the physiological curve for the Pacinian corpuscle does not show a reduction in slope which is the characteristic of the psychophysical curve. By extending the Pacinian psychophysical curve into the low frequencies (dashed line), we can estimate the displacement needed to activate those receptors at low frequencies. The figure shows that, at $25 \mathrm{~Hz}$, a displacement of at least $12 \mathrm{~dB}$ SL (vertical bar) would be necessary in order to activate the Pacinian system. Figure 1 shows that spatial summation at this frequency may be produced at levels between 2 and 5 dB SL (Green \& Craig, 1974; Verrillo, 1974), far 
below those levels that could be expected to activate the receptor.

Our experiments were based on two alternative hypotheses to explain the response of the non-Pacinian population. The first hypothesis suggests that the non-Pacinian receptors may exhibit spatial summation at both threshold and suprathreshold levels of stimulation. Spatial summation produced by the activation of larger numbers of receptors as the contactor size is increased becomes obscured, however, by low-frequency noise within the system, which grows also in direct proportion to the number of units affected. The source of the noise may be attributed to the static displacement of the contactor, muscle dynamics, blood pulsation, and other less well-defined physiologic sources. Since we may expect that both signal and noise grow in direct proportion to each other with contactor size, a consequent improvement in threshold would not be expected.

The Pacinian corpuscle possesses a definite threshold for discharge (Gray \& Sato, 1955) which occurs at fairly high levels of stimulation (Loewenstein \& Mendelson, 1965). Intrinsic noise in the system is virtually nonexistent, providing an excellent background for the detection of signal-induced discharges. Furthermore, the Pacinian corpuscle is relatively insensitive to the low-frequency stimuli assumed to produce noise in the non-Pacinian system. The addition of neural inputs by increasing the size of the contactor may therefore be expected to produce lower thresholds as a consequence of activating more receptors.

Since we were unable to subtract noise from the non-Pacinian channel, we attempted to test our hypothesis by introducing an appropriate background noise to the Pacinian channel. It seemed reasonable that such a procedure would mimic the operating conditions of the non-Pacinian system. The alteration may affect the spatial summation characteristics of these receptors at threshold and result in a nullification of summation effects.

The second hypothesis states that an interaction may occur at suprathreshold levels between the highand low-frequency systems. This does not imply simply that low-frequency spatial summation is the result of an intense stimulation of Pacinian corpuscles. We are suggesting, rather, that when stimuli that activate the systems selectively are delivered simultaneously, the combined neural activity of the systems will produce results not accountable by either system acting alone. Such an interaction could account for low-frequency spatial summation at intensity levels of $12 \mathrm{~dB}$ SL or higher.

The rather complicated set of combinations of noise bandwidth, signal frequency, and contactor size which may reasonably be expected to stimulate

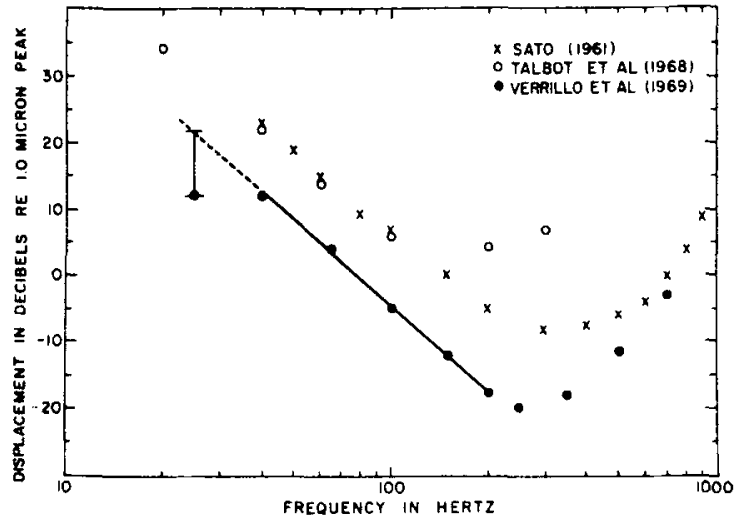

Figure 2. Threshold response of the Pacinian system as a function of stimulus frequency. The solid circles show the psychophysical curve (Verrillo et al., 1969), which becomes flat at low frequencies where the non-Pacinian receptors predominate. The U-shaped portion of the curve is closely paralleled by physiological results. The data of Sato (1961) show the physiological response of individual corpuscles $(x)$ stimulated directly, and those of Talbot et al. (1968) are the responses of corpuscles $(O)$ stimulated by vibrations dellvered to the surface of the skin. Extension of the psychophysical Pacinian curve to low frequencies (dashed line) shows that the displacement necessary to activate Pacinians at $25 \mathrm{~Hz}$ (vertical bar) exceeds the measured threshold by at least $12 \mathrm{~dB}$.

predominantly either one or both of the two receptor systems are summarized here and in Table 1:

(1) Non-Pacinian stimulation: (a) $25-\mathrm{Hz}$ signal in a narrow-band/low masker, using a large or small contactor (Boxes 1 and $1 \mathrm{a})^{1}$; (b) $25-\mathrm{Hz}$ signal in a wide-band masker, using a large or small contactor (Boxes 3 and 3a).

(2) Pacinian stimulation: (a) $250-\mathrm{Hz}$ signal in a narrow-band/high masker, using a large or small contactor (Boxes 5 and $5 \mathrm{a}$ ); (b) $250-\mathrm{Hz}$ signal in a wide-band masker, using a large contactor (Box 6a).

(3) Both systems: (a) $250-\mathrm{Hz}$ signal in a narrowband/low masker, using a large or small contactor (Boxes 4 and 4a); (b) $250-\mathrm{Hz}$ signal in a wide-band masker, using a small contactor (Box 6); (c) $25-\mathrm{Hz}$

Table 1

\begin{tabular}{|c|c|c|c|c|c|}
\hline \multirow[b]{2}{*}{$\begin{array}{c}\text { Signal } \\
(\mathrm{Hz})\end{array}$} & \multicolumn{5}{|c|}{ Masker } \\
\hline & $\begin{array}{c}\text { Contrac- } \\
\text { tor Area } \\
\left(\mathrm{cm}^{2}\right)\end{array}$ & $\begin{array}{c}\text { Narrow } \\
\text { Band } \\
\text { Low }\end{array}$ & $\begin{array}{c}\text { Narrow } \\
\text { Band } \\
\text { High }\end{array}$ & & $\begin{array}{l}\text { Wide } \\
\text { Band }\end{array}$ \\
\hline \multirow{3}{*}{25} & & & 2 & 3 & \\
\hline & .28 & $\overline{\mathrm{p}}^{*}$ & $2 a^{\text {Both }}$ & $3 a$ & $\overline{\mathrm{p}}^{*}$ \\
\hline & 2.9 & $\overline{\mathrm{p}}^{*}$ & Both & & $\overline{\mathrm{p}}^{*}$ \\
\hline \multirow[t]{2}{*}{250} & .28 & Both & ${ }_{5 a}^{5} P^{*}$ & $\begin{array}{l}6 \\
6 a\end{array}$ & Both \\
\hline & 2.9 & Both & P* & & $\mathrm{P}^{*}$ \\
\hline
\end{tabular}

Note $-P=$ Pacinian, $\bar{p}=$ non-Pacinian

$*$ Slope $=1.0 ;$ otherwise slope $=.5$ 


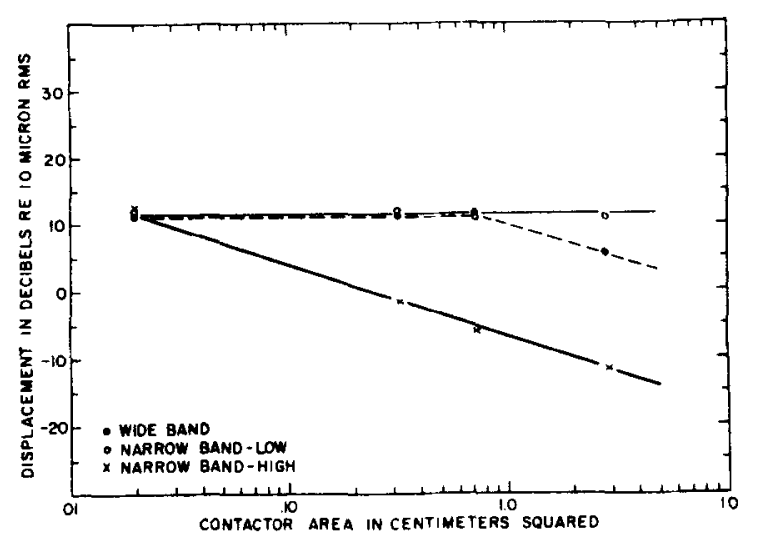

Figure 3. Vibrotactile thresholds as a function of contactor size for three bandwidths of nolse. Narrow band/high $(240-270 \mathrm{~Hz})$, which activates Pacinian corpuscles, summates over space with a slope of $3.0 \mathrm{~dB}$ per doubling of contactor area. Narrow band/low $(15-45 \mathrm{~Hz})$, which activates primarily non-Pacinian receptors. produces no spatial summation. Wide-band noise, which contains frequencies that can activate both types of receptors- produces spatial summation for contactor areas langer than approximately $0.72 \mathrm{~cm}^{2}$.

signal in a narrow-band/high masker, using a large or small contactor (Boxes 2 and 2a).

\section{RESULTS}

A preliminary experiment was performed in order to determine vibrotactile thresholds of detectability as a function of contactor area for the different bandwidths of noise for comparison with sinusoidal data. (Displacement values of the noise were determined by calibrating the voltage output of the accelerometer, at each noise bandwidth, to RMS displacement values measured directly by means of a MTI Fotonic Sensor.) The results (Figure 3) were consistent with data obtained with sinusoidal signals (Verrillo, 1963). The vibration threshold for the narrow-band/high noise ( 240 to $270 \mathrm{~Hz}$ ), plotted as a function of contactor area $(0.02,0.34,0.72$, and $2.9 \mathrm{~cm}^{2}$ ), yielded a slope of approximately $3 \mathrm{~dB}$ per doubling of contactor size, which is the slope produced by a $250-\mathrm{Hz}$ signal. The narrow-band/low noise (15 to $45 \mathrm{~Hz}$ ) resulted in a curve that is flat across all contactor sizes, in agreement with the flat curve of a $25-\mathrm{Hz}$ sinusoid. The wide-band noise ( 2.0 to $4,800 \mathrm{~Hz}$ ) produced a two-limbed curve which is flat to about $0.72 \mathrm{~cm}^{2}$, and for larger contactor sizes the slope decreases at the rate of $3.0 \mathrm{~dB}$ per doubling of area. We may assume, then, that the narrowband/low noise activates primarily the non-Pacinian population; that the Pacinian system is activated by the narrow-band/high noise; and that the wide-band noise is capable of stimulating both systems.

The threshold values obtained with each of the bandwidths of noise are consistent with a duplex theory of vibrotaction; low frequencies stimulate a receptor population that does not produce spatial summation at the threshold of detectability, high frequencies activate a receptor system that summates energy over space. Stimuli containing both high and low frequencies can activate either system, depending on the size of the contactor.

In the main experiments, thresholds were obtained at two frequencies $(25$ and $250 \mathrm{~Hz})$, using two contactor sizes $\left(0.28\right.$ and $\left.2.9 \mathrm{~cm}^{2}\right)$, for each bandwidth of noise. The data were plotted in terms of threshold displacement $(\mathrm{dB})$ as a function of the sensation level of the masker. The effect of masking noise on vibrotactile sensitivity to sinusoids for either contactor size resulted in elevated thresholds that could be approximated by straight lines. At each test frequency, the slope values were dependent upon the combination of contactor size and the frequency components of the masker.

At $25 \mathrm{~Hz}$ (Figure 4), linear relationships between the threshold of the sinusoid and the sensation level of the masker were produced by the following conditions: wide-band masker with the 0.28 - and $2.9-\mathrm{cm}^{2}$ contactors (Boxes 3 and $3 \mathrm{a}$ ) and narrow-band/low masker with both contactors (Boxes 1 and 1a). However, the effect decreased to a slope value of 0.5 when thresholds were measured in the presence of the narrow-band/high masker using either the 0.28 - or $2.9-\mathrm{cm}^{2}$ contactor (Boxes 2 and $2 \mathrm{a}$ ).

At $250 \mathrm{~Hz}$ (Figure 5), the wide-band masker combined with the $2.9-\mathrm{cm}^{2}$ contactor (Box 6a) and the narrow-band/high masker with both the 0.28 - and $2.9-\mathrm{cm}^{2}$ contactors resulted in a slope of 1.0 (Boxes 5 and $5 a$ ). The linear relationship is consistent with expectations for signals detected in the presence of

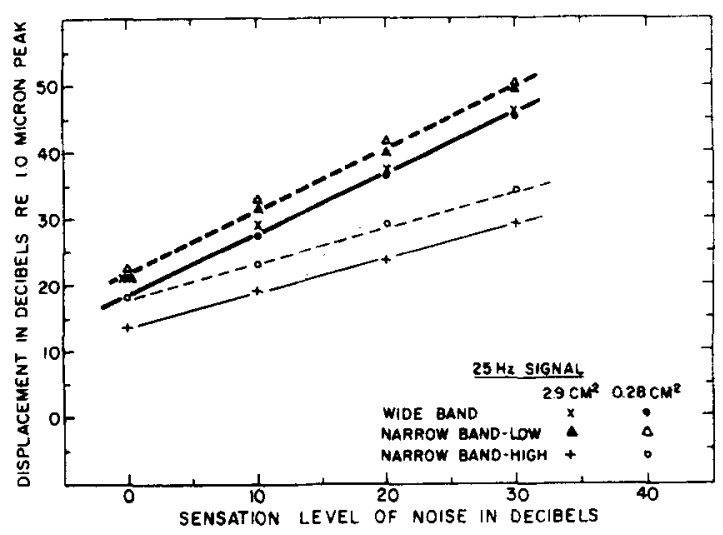

Figure 4. Vibrotactile thresholds of a $25-\mathrm{Hz}$ signal for two contactor sizes, masked by the bandwidths of noise shown in Figure 1. Symbols of the masker-bandwidth/contactor-size combinations are the same as Figure 2. Combinations favoring the activation of the non-Pacinian receptors alone produced a linear effect. Combinations which activated Pacinian and non-Pacinian systems simultaneously produced slopes of 0.5 value. 
increasing levels of masking noise. The slopes decreased to 0.5 , however, when the following conditions were used: wide-band masker with a $0.28-\mathrm{cm}^{2}$ contactor (Box 6) and narrow-band/low masker with both the 0.28 and $2.9 \mathrm{~cm}^{2}$ contactors (Boxes 4 and 4a).

The data of Figures 4 and 5 are replotted in Figures 6 and 7 , respectively. The threshold values of the masker sinusoidal signals are plotted as a function of the absolute intensity of the masking noise referred to 1.0 micron RMS displacement. These figures enable the reader to see the effects of the maskers in absolute rather than relative terms and permit additional comparisons between contactor sizes which are not evident in Figures 4 and 5 . These will be considered in the Discussion section.

It is apparent that the effect of vibratory masking noise on vibrotactile thresholds is a combined function of the signal frequency, the bandwidth of the masker, and the size of the contactor.

\section{DISCUSSION}

We will consider first the possibility that a difference in the signal-to-noise ratios of the cutaneous mechanoreceptor systems may account for the spatial summation produced in the low-frequency system. The attempt to nullify summation effects in the Pacinian system by imposing a lowered signal-to-noise ratio by means of an extrinsic noise was not successful. The anticipated effect would have resulted in the overlap of data points for the two contactor sizes in Figure 5 when the $250-\mathrm{Hz}$ signal was masked either by the narrow-band/high or the narrow-band/low noise. This clearly did not occur. The approximate 15- $\mathrm{dB}$ separation between the two curves is consistent with the summation effect produced by our subjects in the absence of any maskers.

The non-Pacinian system, however, was affected by the narrow-band masking noises. The spatial summation effect observed at high intensities without maskers (Figure 1) for a $25-\mathrm{Hz}$ signal appeared to be nullified by low- or high-frequency narrow-band maskers. This is apparent from the complete overlap of data points in Figures 4 and 6 for the two contactor sizes $\left(2.9 \mathrm{~cm}^{2}, \Delta\right.$, and $\left.0.28 \mathrm{~cm}^{2}, \Delta\right)$. When the curves for the narrow-band/high masker $\left(2.9 \mathrm{~cm}^{2}\right.$, + , and $0.28 \mathrm{~cm}^{2}$, O) are adjusted to absolute masker displacement (Figure 6), there is again no apparent summation in the non-Pacinian population. At suprathreshold levels, this system clearly produces spatial summation in the absence of an extrinsic noise (Verrillo, 1974).

We cannot, at the present time, explain completely the spatial summation effects produced at low signal

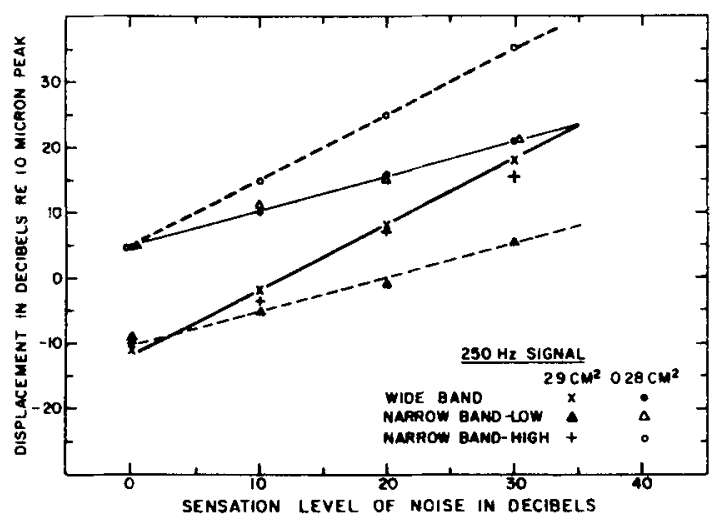

Figure 5. Vibrotactile thresholds of a $250-\mathrm{Hz}$ signal for two contactor sizes, masked by three bandwidths of noise-wide band (2.0-4,800 Hz), narrow band/low $(15-45 \mathrm{~Hz})$, and narrow band/high (240-270 Hz). Symbols refer to comblnations of masker bandwidth and contactor size: wide band, $2.9 \mathrm{~cm}^{2}(x), 0.28 \mathrm{~cm}^{2}(\bullet)$; narrow band/low, $2.9 \mathrm{~cm}^{2}(\Delta), 0.28 \mathrm{~cm}^{2}(\Delta)$; and narrow band/high, $2.9 \mathrm{~cm}^{2}(+), 0.28 \mathrm{~cm}^{2}(O)$. Comblnations favoring the activation of the Pacinian corpuscle system alone produced a linear effect. Combinations which activated Pactilan and non-Pachian receptors simultaneously produced slopes of 0.5 value.

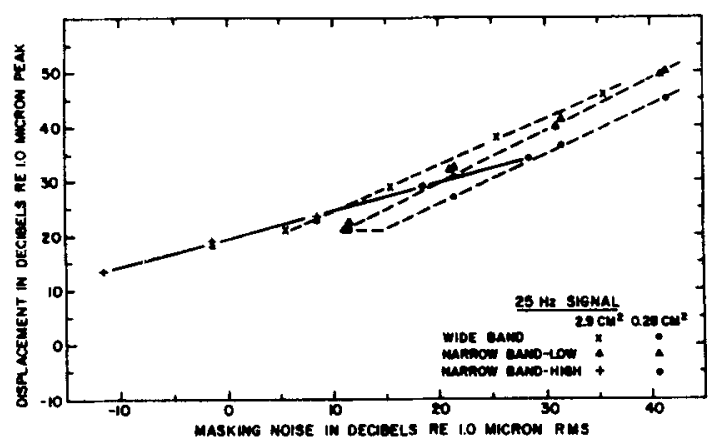

Figure 6. Same as Figure 4, except that the maskmg noise in plotted in absolute terms of decibels referred to 1.0 micron of RMS displacement.

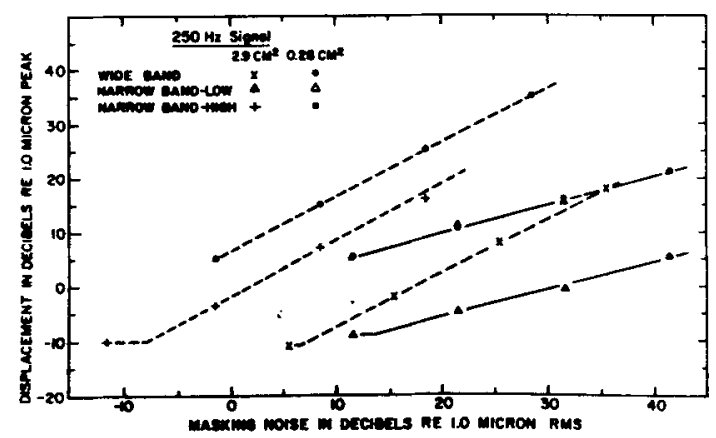

Figure 7. Same as Figure 5, except that the masking nolse b plotted in absolute termin of decibels referred to 1.0 micron of RMS displacement. 
frequencies. The results are equivocal when considered within the context of a signal-to-noise differential between the two systems. Altering the signal-to-noise ratio in the Pacinian system did not affect its capacity to summate energy over space, but the non-Pacinian system failed to show spatial summation when masked by extrinsic narrow-band noise.

Our next consideration concerns the hypothesis that an interaction between the high- and low-frequency systems may explain spatial summation in the low-frequency system. Vibrotactile noise thresholds measured as a function of contactor size (Figure 3) showed that the high-frequency bandwidth produced a spatial summation effect of $3.0 \mathrm{~dB}$ per doubling of area, a characteristic of the Pacinian population. The absence of summation produced by the noise bandwidth containing only low frequencies is characteristic of the non-Pacinian receptor population. The curve of the wide-band noise, which contains energy at low and high frequencies, is flat for small contactor areas and decreases at the rate of $3.0 \mathrm{~dB}$ per doubling of the larger areas. This suggests that, over small areas, the non-Pacinian response dominates when both high and low frequencies are contained in the stimulus. As size increases, the Pacinians dominate the response and spatial summation occurs. Inspection of Table 1 shows that any combination of stimulus parameters that may reasonably be expected to stimulate selectively either the Pacinian population (e.g., $250-\mathrm{Hz}$ signal, narrow-band/high masker) or the non-Pacinian receptor system (e.g., 25-Hz signal, narrow-band/low masker) resulted in a linear relationship between the threshold of detection for sinusoids and the intensity level of the masker. Any condition that results in the combined, simultaneous stimulation of both receptor systems (e.g., $250-\mathrm{Hz}$ signal, narrow-band/low masker) reduced the effect of masking to a 0.5 slope. Increasing the intensity of the masker has less effect when both systems are activated simultaneously, with the noise in one channel and the signal in the other, than when either system alone is activated. A reasonable hypothesis to explain the effect suggests that when both signal and noise are confined to a single channel, they combine linearly at the periphery and produce a linear curve. When signal and noise activate different channels, they add in a nonlinear fashion at a higher level of the nervous system and the resultant masking slope is less than unity. It would be difficult, on the basis of the data, not to conclude that there is an interaction between the two systems at some level of the central nervous system, since a peripheral interaction of signal and masker should produce a slope of 1.0 .
It is difficult to explain the summation effects solely within the context of the operating characteristics of a single receptor system. Although the Pacinian corpuscle can be activated at low frequencies by very intense stimuli (Merzenich \& Harrington, 1969), its potential to explain subjective magnitude functions for low frequencies in terms of its response characteristics alone is limited. Spatial summation at low frequencies begins to occur at relatively low levels of stimulation (Verrillo, 1974), far below those levels that could be expected to activate the receptor (Figure 2). It seems reasonable to suggest that, if spatial summation for low frequencies at suprathreshold levels is the result of an interaction process occurring among receptor systems, the interaction takes place within the central nervous system. The exact nature and locus of the interaction is not known. Studies utilizing contralateral stimuli are being undertaken in order to test this hypothesis. The data also suggest that an alteration of the signal-to-noise ratio of the non-Pacinian system produces a decrease in the spatial summation seen at suprathreshold levels of stimulation.

\section{REFERENCES}

Gray, J. A. B., \& SAto, M. Movement of sodium and other ions in Pacinian corpuscles. Joumal of Physiology (London), 1955. 129, 594-607.

Green, B., \& Craig, J. C. The roles of vibration amplitude and static force in vibrotactile spatial summation. Perception \& Psychophysics, 1974, 16, 503-507.

Harrington, T., \& Merzenich, M. M. Neural coding in the sense of touch: Human sensations of skin indentation compared with the responses of slowly adapting mechanoreceptive afferents innervating the hairy skin of monkeys. Experimental Brain Research, 1970, 10, 251-264.

Johnson, K. O. Reconstruction of population response to a vibratory stimulus in quickly adapting mechanoreceptive afferent fiber population innvervating glabrous skin of the monkey. Journal of Neurophysiology, 1974, 37, 48-72.

Loewenstein, W. R., \& Mendelson, M. Components of receptor adaptation in a Pacinian corpuscle. Journal of Physiology (London), 1965, 41, 377-397.

Merzenich, M. M., \& Harrington, T. The sense of fluttervibration evoked by the stimulation of the hairy skin of primates: Comparison of human sensory capacity with the responses of mechanoreceptive afferents innvervating the hairy skin of monkeys. Experimental Brain Research, 1969, 9, 236-260.

SAto, M. Response of Pacinian corpuscles to sinusoidal vibration. Journal of Physiology, 1961, 159, 391-409.

Talbot, W. H., Darian-Smith, 1., Kornhuber, H. H., \& MountCastle, V. B. The sense of flutter vibration: Comparison of the human capacity with response patterns of mechanoreceptive afferents from the monkey hand. Joumal of Neurophysiology, 1968, 31, 301-334.

VERRILLO, R. T. Effect of contactor area on the vibrotactile threshold. Journal of the Acoustical Society of America, $1963,35,1962-1966$. 
VERRILLo, R. T. Effect of spatial parameters on the vibrotactile threshold. Joumal of Experimental Psychology, 1966, 71, 570-575. (a)

VERRILlo, R. T. Vibrotactile sensitivity and the frequency response of the Pacinian corpuscle. Psychonomic Science, 1966, 4. 135-136. (b)

Verrillo, R. T. Specificity of a cutaneous receptor. Perception \& Psychophysics, 1966, 1, 149-153. (c)

VerRILLO, R. T. A duplex mechanism of mechanoreception. In D. Kenshalo (Ed.), The skin senses. Springfield, Ill: Thomas, 1968.

VERRILLo, R. T. Vibrotactile intensity scaling at several body sites. In F. A. Geldard (Ed.), Cutaneous communication systems and devices. Austin, Tex: Psychonomic Society, 1974.

Verrillo, R. T., Fraioli, A. J., \& Smith, R. L. Sensation magnitude of vibrotactile stimuli. Perception \& Psychophysics, 1969. 6. 366-372.

\section{NOTE}

1. Box numbers refer to Table 1 .

(Received for publication September 18, 1974; accepted April 25, 1975.) 